geschlechtsspezifischer Verfolgung bedrohten oder betroffenen Frauen und Männer, unter denen die Frauen erklärtermaßen die größere Gruppe bilden. Frau Markard sieht dabei genau hin, thematisiert viele Schrecknisse und ordnet sie ein. Erneut ist hier ihre Sprache hervorzuheben, die präzise benennt, aber nicht dramatisiert, und dadurch auch in der Schilderung des Rechtstatsächlichen große Glaubwürdigkeit erzeugt.

Andere Arbeiten zur Genfer Flüchtlingskonvention und zur Qualifizierungs-Richtlinie auf dem Gebiet des Völker- und Europarechts werden sich mit dieser Arbeit auseinandersetzen wollen und müssen. Ihre Rezeption ist auch dadurch begünstigt, dass Frau Markard auf ihrem Gebiet bekannt und schon glänzend vernetzt ist. Sie hat im vergangenen Jahr bereits den Dissertations-Preis der Wilhelm-von-Humboldt-Universität Berlin erhalten, an der die Dissertation entstanden ist. Sie zählt zum Hohenheimer Kreis des Ausländerrechts und ist bereits Mitglied des Netzwerks Migrationsforschung, das einige junge und ambitionierte Rechtswissenschaftlerinnen und Rechtswissenschaftler des öffentlichen Rechts versammelt. Für Aktivitäten zum deutschen Aufenthaltsrecht, aber auch für Einflussmöglichkeiten auf europäische Rechtssetzungsprozesse, an denen der djb sich verstärkt beteiligen will und wird, ist auch dieser rechtspolitische Akzent der Arbeit von nicht zu unterschätzender Bedeutung.

Liebe Frau Markard, in Abwesenheit der Stifterin, Frau Melitta Büchner-Schöpf, ist es meine Aufgabe, Ihnen auch den
Preis zu überreichen. Wie Marie Elisabeth Lüders haben Sie an der Wilhelm-von-Humboldt-Universität in Berlin studiert und promoviert, und wie sie haben Sie für Ihre juristische Dissertation ein Thema mit Gender-Bezug gewählt. Bei Ihnen beiden zeugt auch die Themenwahl von einem sicheren Gespür für Aktualität und von einer klaren rechtspolitischen Absicht. Marie Elisabeth Lüders hat sich zwar dem Wirtschaftsrecht verschrieben und liegt damit weit ab von dem Themenbereich der heute ausgezeichneten Dissertation. Allerdings waren die „im Gewerbe tätigen Frauen und Mädchen“, denen sie ihre Arbeit widmete, um 1910 gewiss nicht sehr verbreitet zu finden, und die Arbeit dürfte - so lässt sich vermuten - an der Universität nicht auf ganz breites Verständnis gestoßen sein. Für das „Gender-Thema“ gilt das in der heutigen Staatsrechtslehre leider immer noch, auch wenn hier Fortschritte zu verzeichnen sind. Schließlich verbindet Sie mit Marie Elisabeth Lüders auch die universitäre Lehre. Während allerdings Frau Lüders die politische Arbeit zum beruflichen Mittelpunkt nahm, sieht es ganz danach aus, dass Sie der Wissenschaft verbunden bleiben werden. Wie sehr Sie dort bereits anerkannt sind, zeigt Ihre Berufung für das kommende Semester zur Vertretung einer Professur für Völker- und Europarecht an der Universität Hamburg.

Im Namen auch meiner Jury-Kolleginnen gratuliere ich Ihnen sehr herzlich zum Preis des Deutschen Juristinnenbundes.

\title{
Marie-Elisabeth-Lüders-Preis 2013: Dank
}

\section{Dr. Nora Markard}

Wissenschaftliche Mitarbeiterin Zentrum für europäische Rechtspolitik (ZERP), Bremen

Liebe Frau Pisal, liebe Frau Prof. Schuler-Harms, liebe Frau Prof. Rudolf, sehr geehrte Kolleginnen, liebe Eltern -

bitte verzeihen Sie mir, wenn ich gleich auf meine Eltern zu sprechen komme, es liegt daran, dass sie heute mit mir hier sind. Dass sie zu Ereignissen wie diesen mitkommen, dabei mitfiebern, ist exemplarisch für die bedingungslose Unterstützung, die sie mir immer haben zukommen lassen. Als ich mit kurzen Haaren und einer sehr cool zerlöcherten Jeans zur Einschulung ging statt mit Kleidchen und Propellerschleife; während meines Studiums und meiner vielen Auslandsaufenthalte; und auch während der Promotionszeit - bis hin zum Korrekturlesen der Dissertation, um das wohl kaum je Eltern herumkommen. Dass ich heute hier stehe, habe ich also in vielerlei Hinsicht ihnen zu verdanken.

Aber dass ich diese Arbeit so schreiben und abschließen konnte, verdanke ich noch vielen weiteren Personen, insbesondere meiner akademischen Familie. Allen voran natürlich meiner Doktormutter, Prof. Dr. Susanne Baer. Sie kann leider heute nicht hier sein, da sie sich an der Yale University mit ihren Kollegen und Kolleginnen von den Verfassungsgerichten der Welt trifft; damit können wir leider nicht mithalten! Susanne Baer ist eine sehr inspirierende Frau, an der man sich auch reiben kann, bei der ich wachsen konnte und die uns viel Raum gelassen hat, neue Ideen zu realisieren - wie zum Beispiel die Humboldt Law Clinic. Zudem ist sie eine Person, die eine quasi magnetische Anziehungskraft hat auf tolle Mitarbeiter/innen und Forscher/ innen, mit denen zu arbeiten mir ein großes Glück war und von denen ich ebenfalls sehr viel gelernt habe und bis heute viel lerne. Es sind zuviele um sie hier alle zu nennen, aber klar ist: Ohne sie hätte ich diese Arbeit so nicht geschrieben. Sie haben mir die Humboldt-Universität zur Heimat gemacht - eine Heimat, die aus feministischer Sicht eng mit dem Namen Marie Elisabeth Lüders verbunden ist.

Heute einen Wissenschaftspreis entgegenzunehmen, der ihren Namen trägt, ist für mich daher eine besonders große Ehre. Marie Elisabeth Lüders war die erste promovierte Frau an einer deutschen Universität, und zwar an meiner Universität, die damals noch Friedrich-Wilhelms-Universität hieß. Eine Frau, die trotz dieser außergewöhnlichen Leistung nie auf die Idee gekommen wäre, sich in den Elfenbeinturm zurückzuziehen. Sie hat sich wissenschafts- und rechtspolitisch engagiert und sich dabei nicht von den Nazis vereinnahmen lassen - sie ist wegen 
ihres feministischen Engagements sogar inhaftiert worden. Nach dem Krieg hat sie die sozialen Strukturen in meiner Heimatstadt Berlin mit aufgebaut. Vor und nach der Naziherrschaft war sie Abgeordnete; ihr prägender Verdienst um die Gleichstellung in der Bundesrepublik ist unschätzbar.

Ihren Namen mit dem Deutschen Juristinnenbund zu verbinden, der diese Arbeit fortsetzt und sich dabei ebenfalls immer auch für die Ausgeschlossenen, auch für Migrantinnen, stark macht, könnte passender nicht sein. Die Arbeit, die die Fachkommissionen des djb, insbesondere die Kommission für die Rechte von Migrantinnen, schätze ich seit vielen Jahren für ihre hervorragende Qualität und ihren rechtspolitischen Anspruch. Heute vor solch exzellenten Kolleginnen zu stehen, ist eine große Ehre.

Mein großer Dank gilt daher heute abend der Stifterin dieses Preises, Frau Dr. Melitta Büchner-Schöpf. Dass sie heute nicht hier sein kann, wie ich eben erfuhr, tut mir sehr leid, und ich hoffe sehr, dass ich bald eine andere Gelegenheit haben werde, ihr auch persönlich meinen Dank auszusprechen. Es ist nicht nur wunderbar, dass sie mit ihrer großzügigen Dotierung des Preises das Andenken an die Namenspatin des Preises ehrt. Frau Dr. Büchner-Schöpf hat sich damit auch entschlossen, die Forschung zur Gleichstellung und zu Geschlechterverhältnissen im Recht zu fördern. Dies ist ein Anliegen, das auch mich antreibt. Die langen Jahre am Lehrstuhl von Susanne Baer haben mich da sehr geprägt und die Themenwahl meiner Dissertation stark beeinflusst. Ursprünglich hat sich das auch im Titel der Arbeit niedergeschlagen, sie ist dann aber deutlich breiter geworden (und auch länger, leider).

Mein Ziel ist es, Geschlechteraspekte zum selbstverständlichen Inhalt guter juristischer Forschung zu machen - ob es nun draufsteht oder nicht. Dass die Jury, der ich hiermit ebenfalls herzlich danken will, diesen Aspekt meiner Arbeit mit diesem Preis heraushebt, meine Intentionen so klar erkannt und so gut herausgearbeitet hat, freut mich besonders. Denn meist geht es ja darum, trotz der Gleichstellungsperspektive gelesen zu werden, und nicht deswegen. Mein Zweitgutachter, der Völkerrechtler Prof. Christian Tomuschat, hat mir denn auch bescheinigt:

„Der feministische Grundansatz, den die Verf. durchweg verfolgt, verführt sie fast nirgendwo zu einer Argumentation, die man als voreingenommen bezeichnen müsste.“

Ja, so soll das sein. Ihre Auszeichnung bestärkt mich darin, diesen Weg weiterzugehen. Vielen Dank.

\section{Pädosexualität und Strafrecht' ${ }^{1}$}

\section{Prof. Dr. Monika Frommel}

Direktorin des Instituts für Sanktionenrecht und Kriminologie der Christian-Albrechts-Universität zu Kiel (1992 bis 2011)

Eine Woche vor der Bundestagswahl veröffentlichte Franz Walter ${ }^{2}$ einen Zwischenbericht zur „pädosexuellen Vergangenheit der Grünen“ (TAZ vom 16.9.2013 „Die fatale Schweigespirale“), der einen Sturm im Wasserglas auslöste und zur Niederlage am 22. September 2013 zumindest mit beigetragen hat. Die Erregung entzündete sich an zwei Ereignissen, einem Beschluss der niedersächsischen Grünen, den Jürgen Trittin 1981 unterschrieben hatte, und einem Text von Volker Beck, der 1988 gedruckt und auf spiegel-online für jeden Interessierten sichtbar war. Der Kontext wurde dabei geflissentlich ignoriert. Vordergründig gelesen gingen sowohl Trittin als auch Volker Beck der damaligen Pädophilen Propaganda auf den Leim. Im genannten Beschluss des Jahres 1981 war von „einverständlicher“, weil „gewaltfreier“ Sexualität zwischen Erwachsenen und Kindern die Rede. Volker Beck ging 1988 noch weiter und erwog in umständlichen Windungen die Entkriminalisierung angeblich unschädlicher pädosexueller Kontakte. Dabei stützte er sich auf die liberale Rechtsgutslehre des Strafrechtswissenschaftlers von Herbert Jäger (1957), welche bis heute anerkannt ist. Sie will - etwas vereinfacht - die Gesetzgebung dazu zwingen, den Schaden, den ein Verhalten nach sich zieht, empirisch zu verifizieren, bevor sie mit Mitteln des Strafrechts kontrollieren darf. Noch nie hat sich die Kriminalpolitik auf diese Forderung eingelassen, auch das BVerfG lässt der Politik mehr Spielraum als diese Doktrin, aber die Idee, man könne den Gesetzgeber dazu zwingen, andere als strafrechtliche Kontrollen zu diskutieren, bevor er straft, schwingt bis heute mit, wenn Strafverschärfungen kritisiert oder Entkriminalisierungen gefordert werden. Theoretisch war seine Position also vertretbar, anrüchig war nur die Annahme, pädosexuelle Kontakte seien nur schädlich, wenn sie gegen den Willen

1 Eine Langfassung des Beitrags ist online: http://www.djb.de/ publikationen/zeitschrift/djbZ-2013-4/djbz-2013-4b6/.

2 Vgl. hierzu die Vorgeschichte: http://blogs.taz.de/hausblog/2013/09/17/ paedophilie-debatte-eine-information-mit-hohem-nachrichtenwert/. Ausführliche Berichte finden sich in der TAZ und SZ am 17. und 18.9.2013, ein besonders sarkastischer in der FAS am 15.9.2013 unter Berufung auf Christian Füller, der bereits den Skandal um die Odenwaldschule zu seinem Thema gemacht hatte, vgl. Christian Füller, Sündenfall, 2010. Der frühere TAZ-Journalist ist zugleich Mitverantwortlicher der Webseite „osodasjahr" und agiert zumindest auch als Sprachrohr der „Opfer“ der Odenwaldschule. Im Gegensatz zur Analyse von Tilman Jens, Freiwild, 2011, ignoriert Christian Füller in seinem Buch die Tatsache, dass verjährte Taten für jede Form der Aufklärung ein ernsthaftes Problem darstellen. Tilman Jens hingegen stellt sich diesem Dilemma und nimmt auch den pädagogischen Anspruch der Schule ernst. Daher analysiert er auf der Basis von mehr als 100 Gesprächen die von ihm konstatierten Parallelwelten der Odenwaldschule zur Zeit der Übergriffe: Er zeigt den Traum vom pädagogischen „Paradies“ auf der einen und die durchlittene „Hölle“ derer, die missbraucht wurden und in der Spirale des Schweigens und Verschweigens untergingen. Er beobachtet aber auch kritisch, was Christian Füller für legitim hält: Eine „Aufklärung“, die in eine Hetzjagd umkippt, einen massenhaften „Missbrauch durch den Missbrauch“. Ohne Anhörung der Betroffenen werden deren längst verjährte Taten von indirekt Betroffenen „aufgedeckt“ und unkontrolliert „,veröffentlicht“. Wer sich empören will, sollte sich über beides empören. 